\title{
Tientos antropológicos sobre la pandemia
}

EVANGEIDOU, S.; MARTÍNEZ-HERNÁEZ, A. (Eds.)

RESET - Reflexiones antropológicas ante la pandemia de covid-19

Tarragona: Publicacions Universitat Rovira i Virgili, 2020

\section{| ${ }^{1}$ Francisco Ortega |}

1 Instituto de Medicina Social, Universidade do Estado do Rio de Janeiro. Rio de Janeiro-RJ, Brasil (fjortega2@gmail.com). ORCID: 0000-0002-5039-9449

El Medical Anthropology Research Center de la Universitat Rovira i Virgili de Cataluña ${ }^{1}$ publica hace una década una interesantísima colección de libros de Antropología Médica. ${ }^{2}$ Todos los libros son de acceso libre. El libro que nos ocupa aquí es el último título de la colección, RESET - Reflexiones antropológicas ante la pandemia de Covid-19, editado por la psicóloga Stella Evangelidou y el antropólogo Angel Martínez-Hernáez. ${ }^{3}$ RESET es un libro extremamente original, escrito de forma coral desde la experiencia del RESET, definido por los editores como "la sensación de reinicio, paréntesis o stand-by que ha sacudido nuestras vidas desde el inicio del año 2020 a consecuencia de la pandemia de Covid-19” (p. 11). El volumen incorpora 48 reflexiones breves (con excepción del primer y el último texto, el resto tienen una extensión media de 2 páginas), elaboradas durante el punto álgido de la pandemia, entre el 14 de abril y el 11 de mayo de 2020 en diferentes países. La mayoría de las contribuciones son en castellano, aunque el volumen incorpora también algunos textos en catalán, portugués, inglés e italiano.

RESET se une a la serie de reflexiones (auto)etnográficas que hemos visto surgir desde el inicio de la pandemia, y que incluyen, sin querer ser exhaustivo, la 
serie "Dispatches from the pandemic" del sitio web interdiciplinar Somatosphere $;^{4}$ "The Corona Diaries auto-ethnography Project", publicado por Curare. Journal of Medical Anthropology; ${ }^{5}$ los "Corona Diaries" del departamento de sociología de la Universidad de Lund; ${ }^{6}$ los "Pandemic Diaries" de la American Ethnological Society; el numero especial dedicado a la pandemia de la revista Social Anthropology, ${ }^{8}$ y en Brasil la serie sobre "Cientistas Sociais e o coronavirus" publicada por la Associação Nacional de Pós-Graduação e Pesquisa em Ciências Sociais (ANPOCS). ${ }^{9}$

RESET es un libro que incorpora una multitud de temas y de perspectivas que los editores han organizado de manera inteligente en 6 secciones: miradas (desde casa, desde el balcón), atención (reflexiones sobre los sistemas sanitarios y autoetnografía de los profesionales de salud), relaciones (consecuencias sociales del confinamiento), vulnerabilidades (desigualdades sociales en la pandemia) metodologías (reflexión sobre las metodologías antropológicas contemporáneas) y propuestas (de cambios en salud pública, educación para salud, modelos de curación). A pesar de esta organización, diversos temas y textos se superponen en las diferentes secciones del libro.

Escribir una reseña que englobe todos los textos y temas abordados en este libro es prácticamente imposible. En estas páginas voy a concentrarme en algunos temas que no necesariamente corresponden a las secciones del libro pero que circulan entre varios textos y tienen una cierta resonancia con cuestiones que me parecen cruciales en relación con la pandemia de Covid-19, y que me gustaría colocar en dialogo con diferentes capítulos del libro.

El primer punto se refiere a las políticas de salud global promovidas por la Organización Mundial de la Salud (OMS) necesarias para "aplanar la curva" de la pandemia, basadas en lockdowns indiscriminados, a mpliación de pruebas diagnósticas y atención hospitalaria para los casos graves. Como diversos comentadores han observado, esas políticas verticales (top-down) y de "tamaño único" (one size fits all), no son factibles en muchos países del sur global y pueden provocar una crisis económica sin precedentes, el aumento de las desigualdades raciales, de género y económicas, así como las enfermedades crónicas, y la violencia doméstica y de género (CASH; PATEL, 2020).

Frente a políticas globales de tamaño único, especialistas en salud pública han demandado "alternativas realistas" adecuadas a contextos de extrema vulnerabilidad que consideren las condiciones de vida de esos individuos y comunidades (GLASSMAN; CHALKIDOU; SULLIVAN, 2020). Esas medidas realistas deben 
comenzar por proteger la salud pública básica, mejorar las condiciones de acceso a agua y a saneamiento, garantizar la seguridad alimentaria y transferencias de renta que permitan que las personas se queden en casa. En sintonía con esta posición, un editorial de Lancet recomienda que cada región identifique y dé soporte a los grupos considerados más vulnerables para evitar el agravamiento de la pandemia y la ampliación de las desigualdades en salud (EDITORIAL, 2020).

En el libro RESET son sobre todo los textos sobre América Latina los que colocan esa cuestión de forma más preeminente. Marcelo Sarlingo alerta que la pandemia se ceba con los territorios de precariedad habitacional en las "villas miserias" de Buenos Aires, caracterizadas por la falta de agua potable (p. 105), situación criticada también por Alejandro Avalos, que observa que en Argentina el "ciudadano tipo" o una "familia tipo" que se puede permitir quedarse en casa, no solo homogeniza la población, sino que ignora la situación de toda la parcela de la población en situación de pobreza (p.140). El texto de Karol Veiga Cabral y el de Marcio Mariath y Adriele Cardoso examinan la situación de extrema precariedad del Estado de Pará en el norte de Brasil, donde menos del 50\% de la población tiene acceso a agua potable. Mariath y Cardoso se preguntan: "Como fazer isolamento social para famílias inteiras que vivem amontadas em um pequeno cômodo? Como higienizarse e descontaminar-se sem saneamento básico e sequer água para beber?” (p. 148). Una situación semejante a la descrita en el texto de Lorenzo Juárez, David Conde y Borja Rivero sobre inseguridad alimentaria y Covid-19 en Guatemala, que enfatiza también la dificultad de realizar higiene personal para quien no tiene agua potable, o mantener el aislamiento físico en las atestadas pickups usadas para el desplazamiento en el país (p. 166).

Sin embargo, no estamos hablando de una situación exclusiva del llamado Sur Global. En el caso de España, esta situación de vulnerabilidad afecta en particular, como explica Stella Evangelidou, a las familias expuestas a la violencia doméstica y de género, a los individuos con discapacidad o con trastornos metales entre otros colectivos (p.67). Antoniu Llort también examina la situación de vulnerabilidad vivida por diversos "outsiders" en el país, que viven sin ingresos y vivienda fija, como usuarios de servicios de adicciones, personas que han pasado por la cárcel, enfermos de SIDA o hepatitis (p.151), y Raluca Cosmina nos habla de los individuos sin hogar (p.156). Los textos mencionados nos recuerdan la necesidad de dirigir la mirada a a la diversidad de los contextos, llevando en cuenta las realidades locales y 
las condiciones de vida de individuos y comunidades. Ese tema es fundamental para una discusión antropológica de la pandemia.

Otro tema correlato que aparece en diversos textos del volumen nos remite a la organización de redes de solidaridad y ayuda mutua en la pandemia. En el caso de Brasil favelas en las grandes ciudades brasileñas se han organizado para ocupar el vacío de una presencia estatal limitada. El hastag \#COVID19NasFavelas ha sido usado por habitantes de favelas en Rio de Janeiro para denunciar la desigualdad social acentuada durante la pandemia, el estado de excepción vivido por muchas comunidades y la "necropolítica" del estado brasileño. Junto a estas reivindicaciones más generales hay otras más específicas sobre la falta de acceso a pruebas de Covid-19 y, la falta de camas de hospital y de UCI, el subregistro de casos y la interrupción de la distribución de comida por causa de las operaciones policiales (LIMA, 2020).

Las diferentes iniciativas comunitarias en desarrollo por diferentes grupos en Brasil y en otros lugares del Sur Global no se pueden reducir a actividades de caridad; tampoco están simplemente llenando localmente y de forma precaria el vacío dejado por la ausencia estatal (ORTEGA; ORSINI, 2020a; ORTEGA; BEHAGUE, 2020). Diferentes activistas están desafiando los puntos de vista convencionales y críticos de la participación comunitaria, ya que están comprometidos en una forma de gobernanza colectiva que funciona de manera sinérgica y simultánea a corto plazo (vertical) y a largo plazo (horizontal). Activistas y líderes comunitarios están trabajando para contener la pandemia al mismo tiempo que abordan las condiciones de vivienda, la seguridad alimentaria, la salud mental, la violencia doméstica y de género y la represión policial (ORTEGA; BEHAGUE, 2020). No se debe subestimar la importancia de esas acciones. Un informe del Instituto Polís, una ONG brasileña, concluye que la favela de Paraisópolis de São Paulo consigue controlar la pandemia mejor que el municipio de São Paulo. Las medidas adoptadas en la comunidad abarcan la creación del sistema de "presidentes da rua", compuesto por más de 400 voluntarios responsables de monitorear a las familias, recolectar y distribuir cestas de alimentos, y combatir fake news. La comunidad también contrató ambulancias y convirtió escuelas en centros de aislamiento (POLIS, 2020). El informe enfatiza que Paraisópolis puede servir de modelo para otras comunidades de Brasil y del Norte Global. Al mismo tiempo iniciativas como esa desafían orientaciones básicas de la salud global, que presuponen que las intervenciones se conciben en el Norte Global y son implementadas en el sur 
Global por profesionales capacitados por representantes de las iniciativas globales (DALGISH, 2020; ORTEGA; BEHAGUE, 2020; SHAMASUNDER et al. 2020).

En el libro RESET ese tema aparece principalmente en los textos referentes a la situación en América Latina. Sobre el contexto de México, Eduardo Menéndez nos habla de las posibilidades de organización y movilización de los sectores marginales, las personas sin trabajo, las mujeres que sufren violencia de género, y los grupos indígenas (p.19). Marcio Mariath y Adriele Cardoso escriben sobre la situación del estado brasileño de Pará y la necesidad de construir con los agentes comunitarios de salud "formas singulares de enfrentamiento de la pandemia" que consideren tanto "directrices sanitarias" como "saberes profanos" (p. 148). Raluca Cosmina Budian, nos introduce a la lucha contra el "sinhogarismo" que realiza en España la Federación Europea de Organizaciones Nacionales que Trabajan con el Sinhogarismo (Feantsa) (p. 156), y la antropóloga y enfermera en un hospital un hospital universitario comarcal de Barcelona Rita Prat nos recuerda los gestos de solidaridad y compañerismo que no suelen darse en condiciones de "normalidad" en las unidades de cuidados intensivos (p. 62).

La participación comunitaria en el diseño e implementación de políticas e intervenciones sensibles localmente es un elemento fundamental para asegurar su eficacia de control de la pandemia en contextos de extrema vulnerabilidad. Por ejemplo, estudios etnográficos realizados en Uganda y otros países de África subsahariana muestran que las experiencias de la lucha contra el ébola y el VIH en el continente africano pueden servir de inspiración para la actual pandemia, pues conceden un papel central a las comunidades (DU TOIT, 2020; JOHNSON; GORONGA, 2020). Las experiencias previas de contención de estas enfermedades enseñan que los enfoques basados en intervenciones verticales e imposición de lockdowns radicales, los cuales con frecuencia involucran el uso de violencia policial y militar, son poco efectivos, y pueden provocar diversas formas de resistencia comunitaria. Comunidades son realidades complejas y únicamente sus miembros pueden informar a los profesionales de salud pública sobre su realidad concreta y lo que funcionará para contenerla en función de sus contextos locales (JOHNSON; GORONGA, 2020). La situación vivida por refugiados en diversos lugares del Sur Global también ha redundado en su participación en tareas de información, capacitación, soporte legal entre otras actividades. Las respuestas localizadas, diseñadas e implementadas por refugiados para refugiados, ponen de manifiesto como los propios individuos sobre los que 
responden de manera más efectiva a las crisis sanitarias que afectan a sus comunidades (ALIO et al., 2020). Estudios etnográficos en el campo de la antropología médica han constatado repetidamente la importancia de la participación comunitaria en la promoción de intervenciones eficaces en diversas pandemias, algo que las iniciativas de salud global insisten en ignorar.

En el libro RESET el texto de Almudena Mari-Sáez destaca la participación comunitaria a partir del ejemplo de la epidemia de ébola en África occidental. Irene Pérez y Maria Jesus Girona nos hablan del trabajo de médicos del mundo en la intervención de la Covid-19 en contextos internacionales y subrayan que las comunidades deben formar parte del proceso de toma de decisiones: "Toda la comunidad debe estar presente en la discusión y mantenerse informada de los pasos que hay que seguir, así como de los porqués y paraqués" (p. 171). El texto de Jacinto López también nos recuerda cómo las experiencias en epidemias anteriores han demostrado que el trabajo de participación comunitaria es vital.

Un informe reciente de la Regional Network for equitiy in health in East and Southern Africa (EQUINET) y el Shaping health consortium llamado: "we are subjects, not objects in health: Communities taking action in Covid-19" justamente nos remite al texto de Ángel Martínez Hernáez que nos describe el trabajo antropológico como el "reconocimiento de los sujetos como sujetos y las cosas como cosas", sujetos que se "cuidan, se curan. También se odian y se matan, claro. Pero se reconocen como sujetos incluso en esos momentos" (p. 28). El informe de EQUINET relata 42 experiencias de participación comunitaria en diversas regiones del planeta y su mensaje principal se resume en la frase siguiente: "La respuesta al Covid no necesita generar miedo y coacción. Puede ser inclusiva, creativa, equitativa y participativa. La coproducción y la codeterminación con las comunidades afectadas no son un 'complemento' opcional de las respuestas al Covid-19" (EQUINET, 2020).

Otro tema que me parece muy relevante y que varios textos del libro examinan concierne la importancia de la atención primaria en el combate al coronavirus. Josep Comelles examina cómo la pandemia ha mostrado la fragilidad del modelo hospitalocéntrico. Enrique Perdiguero-Gil va en la misma línea, y vincula el hospitalocentrismo y el desprestigio de la Atención Primaria al modelo biologicista dominante focalizado en el virus y en las vacunas. Vicente Rabanaque y María Moreno Rubio evocan el trabajo fundamental de la atención primaria, una "gran gesta" que "rara vez es noticia" y que "supone toda una trayectoria profesional 
volcada al conocimiento experiencial, personal y directo, que proporciona una mirada holística y longitudinal que hace posible escuchar, acompañar, atender y entender, desde la confianza fraguada durante los años, un relato e incluso toda una narrativa vital, a veces familiar" (p. 42)

La importancia de la atención primaria y su situación de desmonte, precariedad y subfinanciación en diversos países constituye un desafío global que la pandemia ha puesto de manifiesto de forma dramática. En Rio de Janeiro el alcalde Marcelo Crivella realizó un corte significativo de gastos en atención primaria, provocando el desmonte de la Estratégia de Saúde da Família, dimisiones en masa de profesionales y agentes comunitarios y una política deliberada de desinformación ciudadana (FERNANDES; ORTEGA, 2020). No resulta por lo tanto sorprendente que a inicios de septiembre la ciudad de Rio de Janeiro superó las 148,5 muertes por 100 mil habitantes, y que le da a la ciudad el récord del peor índice del mundo (BARREIRA, 2020). Por otro lado, el trabajo de la atención primaria puede ser ejemplar, como el caso de un centro de atención primaria de salud en el Morro da Conceição en Recife, en el nordeste de Brasil, que asumió el liderazgo en el desarrollo de intervenciones adaptadas a la cultura, cambiando su proceso de trabajo y mejorando las capacidades de telesalud y monitoreo remoto para convertirse en un ejemplo de prestación de atención en el país (FERNANDES; PACHECO; FERNANDEZ, 2020). Como dice una de las autoras del artículo publicado en New England Journal of Medicine que tiene como base esa intervención: "O editor de uma renomada revista do mundo simplesmente não conseguia entender como fazemos tanto com tão pouco" (COMUNICACIÓN PERSONAL). "Hacer tanto con tan poco" define muchas soluciones creativas del Sur Global en el contexto de la pandemia.

Entre los múltiples temas que aparecen en el libro me parece importante resaltar la cuestión de la verdad científica. El texto de Enrique Perdiguero habla de la “aceptación acrítica de la llamada 'evidencia científica' ha militado en contra de una gestión eficiente de la cuestión” (p. 46). Esa es sin duda una consideración importante, pero para un reseñista que vive en Brasil el problema es posiblemente el contrario, pues la gobernanza del Covid-19 en este país se caracteriza por un "neoliberalismo autoritario" que convive con lo que el médico y antropólogo Vinh-Kim Nguyen ha denominado de "gobierno por excepción" (NGUYEN, 2009), caracterizado por la ausencia o presencia limitada del Estado. En el país sudamericano neoliberalismo autoritario y gobierno por excepción se alían a la 
movilización estratégica de la ignorancia (McGOEY, 2012) y al rechazo o negación del "conocimiento incómodo" (RAYNER, 2012), que no cede a los deseos del presidente en su promoción de curas milagrosas, diseminación de fake news y negacionismo científico (ORTEGA; ORSINI, 2020a).

La pandemia ha sido y continúa siendo politizada en numerosos países del Norte y Sur global. Diversos artículos han examinado el uso político realizado por diversos líderes mundiales de la crisis Covid-19 para imponer medidas draconianas, silenciar a los críticos y asegurar su base de poder. Entre los ejemplos más prominentes encontramos los del primer ministro húngaro Viktor Orbán, que declaró un estado de emergencia que le permite consolidarse y gobernar por decreto, del presidente de Filipinas, Rodrigo Duterte, que se otorgó poderes de emergencia con la justificativa de controlar la difusión de fake-news, del primer ministro israelí, Benjamin Netanyahu, que usó el coronavirus para suspender los tribunales que le acusaban de corrupción, de los gobiernos de Argelia e India, que han usado la pandemia para reprimir manifestaciones y minorías étnicas, o de los de China, Corea del Sur y Rusia que utilizaron la pandemia Covid-19 para fortalecer la vigilancia digital (ROTH, 2020; ORTEGA; ORSINI, 2020b). Todos comparten un estilo de politización pandémica que no niega la ciencia; más bien, moviliza evidencia científica para justificar y aplicar enfoques punitivos para controlar la población.

Encontramos la politización de la pandemia en el texto de Nicolás Morales sobre la crisis sanitaria y la "guerra" contra el coronavirus en Chile. El presidente Sebastián Piñera emplea la alegoría del "enemigo poderoso" para asociar el coronavirus con el propio pueblo movilizado contra las desigualdades socioeconómicas generadas por las políticas neoliberales sacralizadas en la Constitución de 1980 y profundizadas durante la posdictadura, y con eso declarar la guerra a los dos enemigos (p.179). Sentí falta de más textos que reflexionasen sobre el tema de la politización, a pesar de que el tema está presente en diversos capítulos: Jiun Isabel Zhang Yim nos ofrece una bella reflexión autoetnográfica sobre estigmatización y xenofobia en tiempos de pandemia (p. 86); la lógica de construcción del "cittadino responsabile" aparece en el texto de Valentina Sbocchia, un individuo caracterizado por la obligación moral de "seguire le norme per non infettare sé stesso e l'intera comunità, una logica che diffonde un'idea abbastanza perversa di colpevolizzazione e di vulnerabilità" (p. 90); y Elisa Alegre-Agís nos habla de los "malos ciudadanos" que no asumen su responsabilidad, y que aunque muchas veces son los más vulnerables (como en el 
caso de los autistas), son, sin embargo, culpabilizados y abucheados por el "policía del balcón” de turno (p. 97).

En fin, tenemos en manos un bello repertorio de reflexiones antropológicas escritas desde el RESET, el stand-by que posibilita un nuevo comienzo, y que, como dicen los editores de está excelente colección, "alimenta el sueño de una posible parada y reparación de la globalización y sus disrupciones, como el impacto de la acción humana en el cambio climático, las inequidades en la salud, la pobreza, la discriminación y el racismo, la mercantilización de la vida y las violaciones sistemáticas de los derechos humanos, entre otras muchas" (p. 11). El libro es una importante contribución en esa dirección.

\section{Referencias}

ALIO, M. et al. By refugees, for refugees: refugee leadership during Covid-19, and beyond. Forced Migration Review, 64, Junio, p. 76-78, 2020. https://www.fmreview.org/issue64/alioalrihawi-milner-noor-wazefadost-zigashane

BARREIRA, G. Com 10 mil mortes por Covid, se fosse um país, Rio seria líder em óbitos por 100 mil habitantes. G1 O Globo. 10 sept. 2020. Disponible: <https:/g1.globo.com/rj/rio-dejaneiro/noticia/2020/09/10/com-10-mil-mortes-por-covid-se-fosse-um-pais-rio-seria-lider-emobitos-por-100-mil-habitantes.ghtml>. Acceso en: 20 oct. 2020.

CASH, R.; PATEL, V. Has COVID-19 subverted global health? Lancet, v. 395, n. 10238, p. 1687-1688, 2020. https://doi.org/10.1016/S0140-6736(20)31089-8

DALGLISH, S. L. Covid-19 gives the lie to global health expertise. Lancet, 26 mar. 2020. pii: S0140-6736(20)30739-X. Doi: 10.1016/S0140-6736(20)30739-X.

DU TOIT, A. COVID-19 responses in Africa: Ok, one size doesn't fit all. Now what? African Arguments. 28 abril. 2020. https://africanarguments.org/2020/04/28/covid-19-responses-inafrica-ok-one-size-doesnt-fit-all-now-what/

EDITORIAL. Redefining vulnerability in the era of Covid-19. Lancet, 395, n. 10230, p. 1089, 4 abr. 2020. doi.org/10.1016/S0140 - 6736(20)30757-1

EQUINET. We are subjects, not objects in health: Communities taking action in Covid-19. Regional Network for equitiy in health in East and Southern Africa (EQUINET) and Shaping health consortium. 30 sept. 2020. https://reliefweb.int/sites/reliefweb.int/files/resources/EQ\%20 Community\%20Engagement\%20synthesis\%20rep\%20Sep2020.pdf Acceso en: 20 oct. 2020.

FERNANDES, L.; ORTEGA, F. A Atenção Primária no Rio de Janeiro em tempos de Covid-19. Physis. Revista de Saúde Coletiva, v. 30, n. 3, p. e300309, 2020. http://dx.doi.org/10.1590/ s0103-73312020300309 
FERNANDES, L.; PACHECO, R. A.; FERNANDEZ, M. How a Primary Health Care Clinic in Brazil Faces Coronavirus Treatment within a Vulnerable Community: The Experience of the Morro da Conceição area in Recife. Nejm Catalyst Innovations in Care Delivery, 10.1056/ CAT.20.0466, 2020. https://doi.org/10.1056/CAT.20.0466

Glassman, A.; CHAlKIDOU, K.; SUllivan, R. Does One Size Fit All? Realistic Alternatives for COVID-19 Response in Low-Income Countries. Center for Global Development. 2 abr. 2020. <https://www.cgdev.org/blog/does-one-size-fit-all-realistic-alternatives-covid-19response-low-income-countries>. Accesso en: 12 abr. 2020

JOHNSON, O.; GORONGA, T. Why communities must be at the centre of the Coronavirus disease 2019 response: Lessons from Ebola and human immunodeficiency virus in Africa. African Journal of Primary Health Care \& Family Medicine, v. 12, n. 1, 2020. doi:https:// doi.org/10.4102/phcfm.v12i1.2496

LIMA, T. DozeEvidências da Necropolítica Frenteà Covid-19 nas Favelas\#OQueDizemAsRedes. RioOnWatch. \#Covid19NasFavelas. 12 mayo 2020. https://rioonwatch.org.br/?p=47191 Acceso: 15 sept. 2020.

McGOEY, L. Strategic unknowns: Towards a sociology of ignorance. Economy and Society, v. 41, n. 1, p. 1-16, 2012. https://doi.org/10.1080/03085147.2011.637330

NGUYEN, V.-K. Government-by-exception: Enrolment and experimentality in mass HIV treatment programmes in Africa. Social Theory \& Health, v. 7, n. 3, p. 196-217, 2009. https:// doi.org/10.1057/sth.2009.12

ORTEGA, F.; BEHAGUE, D. O que a medicina social latino-americana pode contribuir para os debates globais sobre as políticas da Covid-19: liçóes do Brasil. Physis. Revista de Saúde Coletiva, v. 30, n. 2, p. e300205. Epub June 26, 2020. https://dx.doi.org/10.1590/s0103-73312020300205

ORTEGA, F.; ORSINI, M. Governing COVID in Brazil: Dissecting the Ableist and Reluctant Authoritarian. Somatosphere. Series: Dispatches from the Pandemic. 17 ab. 2020b. Disponible: <http://somatosphere.net/2020/governing-covid-in-brazil-dissecting-the-ableist-and-reluctantauthoritarian.html/>. Acceso en: 18 abr. 2020.

ORTEGA, F.; ORSINI, M. Governing COVID-19 without government in Brazil: Ignorance, neoliberal authoritarianism, and the collapse of public health leadership. Global Public Health, v. 15, n. 9, p. 1257-1277, 2020a. DOI: 10.1080/17441692.2020.1795223

PÓLIS. Paraisópolis tem melhor controle da pandemia do que o município de São Paulo. Report. Instituto Pólis. Junio, 2020 https://agenciagalo.com/wp-content/uploads/2020/06/ Release-P\%C3\%B3lis-Parais\%C3\%B3polis.pdf . Acceso en: 18 ago. 2020.

RAYNER, S. Uncomfortable knowledge: The social construction of ignorance in science and environmental policy discourses. Economy and Society, v. 41, n. 1, p. 107-125, 2012. https://doi. org/10.1080/03085147.2011.637335 
ROTH, K. How Authoritarians Are Exploiting the Covid-19 Crisis to Grab Power. The New York Review of Books, 31 mar. 2020. Disponible en: https://www.nybooks.com/daily/2020/03/31/ how-authoritarians-are-exploiting-the-covid-19-crisis-to-grab-power/. Acceso en: 20 abr. 2020. SHAMASUNDER, S. et al. COVID-19 reveals weak health systems by design: Why we must re-make global health in this historic moment. Glob Public Health, v. 15, n. 7, p. 1083-1089, 2020. DOI: $10.1080 / 17441692.2020 .1760915$

\section{Notas}

${ }^{1}$ http://www.marc.urv.cat/es

${ }^{2}$ http://www.publicacionsurv.cat/llibres-digitals/antropologia-medica.

${ }^{3}$ http://digital.publicacionsurv.cat/index.php/purv/catalog/view/448/465/1046-1

${ }^{4}$ http://somatosphere.net/2020/covid-19-forum-introduction.html

${ }^{5}$ https://boasblogs.org/witnessingcorona/curare-corona-diaries-project

${ }^{6}$ https://www.soc.lu.se/en/research/research-projects/corona-diaries-0

${ }^{7}$ https://americanethnologist.org/features/pandemic-diaries

${ }^{8}$ https://onlinelibrary.wiley.com/toc/14698676/2020/28/2

${ }^{9} \mathrm{http}: / / \mathrm{www}$.anpocs.com/index.php/ciencias-sociais/destaques/2325-boletim-semanal 